\title{
COMMENTARIES
}

\section{Improving End-of-Life Care for People with Dementia in LTC Homes During the COVID-19 Pandemic and Beyond}

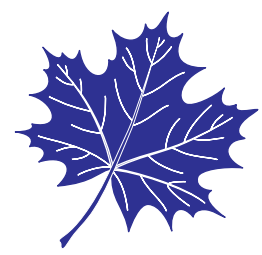

Sharon Kaasalainen, $\mathrm{PhD}^{1}$, Lynn Mccleary, $\mathrm{PhD}^{2}$, Shirin Vellani, MN-NP${ }^{3}$, Jose Pereira, MBChB, CCFP(PC), MSc, FCFP 4,5

${ }^{1}$ School of Nursing, McMaster University, Hamilton; ${ }^{2}$ Faculty of Applied Health Sciences, Nursing, Brock University, St. Catharines; ${ }^{3}$ KITE-Toronto Rehabilitation Institute, University Health Network, Toronto; ${ }^{4}$ Division of Palliative Care, Department of Family Medicine, McMaster University, Hamilton; ${ }^{5}$ Pallium Canada, Ottawa, ON

https://doi.org/10.5770/cgj.24.493

\begin{abstract}
COVID-19 pandemic has resulted in a significant increase in deaths in long-term care homes (LTCH). People with dementia living in LTCHs represent one of the most frail and marginalized populations in Canada. The surge of COVID-19 cases in LTCHs and rationing of health-care resources during the pandemic have amplified the pre-existing need for improvements in palliative and end-of-life care in LTCHs. This position statement, created by a task force commissioned by the Alzheimer Society of Canada, provides recommendations for a multipronged coordinated approach to improving palliative and end-of-life care of people with dementia living in LTCHs during the COVID-19 pandemic and beyond.
\end{abstract}

Key words: dementia, COVID-19, palliative approach, endof-life, long-term care

\section{BACKGROUND}

The devastating impact of the COVID-19 pandemic has resulted in a significant increase in deaths in long-term care homes (LTCH). ${ }^{(1)}$ With some exceptions, many LTCHs have not implemented a palliative approach, ${ }^{(2)}$ and the LTCH workforce is generally ill-prepared to provide this approach. As a result, there has been an excessive reliance on invasive medical care at end-of-life (EOL), unnecessary hospital transfers, and many people are denied opportunities to participate in their EOL planning, especially earlier in the illness when they still have capacity to do so. ${ }^{(3-5)}$ These issues have been magnified during the COVID-19 pandemic. Families are required to make important decisions without preparatory engagement, and health-care providers in LTCHs have to initiate advance care planning (ACP) and EOL discussions without essential competencies. The surge of COVID-19 cases in LTCHs and rationing of health-care resources during the current pandemic ${ }^{(6,7)}$ have amplified the pre-existing need for improvements in palliative and EOL care in LTCHs.

This statement provides key recommendations to improve the care of people with dementia living in LTCHs during the COVID-19 pandemic and beyond. Recommendations are listed in Table 1. Details about the recommendations are provided below.

\section{LTCHs Should Implement a Palliative Approach to Dementia Care}

The Quality End-of-Life Care Coalition of Canada endorsed a national framework for a palliative approach to the care of persons with chronic, progressive, life-limiting illnesses, including dementia. This includes initiating a palliative care approach early in the illness trajectory alongside chronic

TABLE 1.

Recommendations for palliative and EOL care of people with dementia in LTCHs

$1 \quad$ Long term care homes should implement a palliative approach to dementia care

2 People with dementia should be engaged in advance care planning early

3 Family members and significant others should be prepared and supported for end-of-life decision making

$4 \quad$ End of life symptoms and psychosocial and spiritual needs should be managed effectively

5 After death care should be provided consistently with local public health guidelines and evidence about SARSCoV-2 transmission.

6 Grief and bereavement support should be provided to family members of deceased residents, LTC home staff, and surviving LTC home residents, including residents with dementia. 
disease management, with a seamless transition to appropriate EOL planning and care. ${ }^{(8,9)}$ To integrate a palliative approach with dementia care in $\mathrm{LTCH}$, we recommend three overarching strategies:

a) adopting the palliative approach in the whole facility;

b) educating and training the LTCH workforce; and

c) adopting tools and policies that enhance care and support staff, to implement the palliative approach. ${ }^{(10,11)}$

Integrating a palliative approach starts with leadership teams embracing and intentionally supporting it, ensuring the right conditions are put in place for its adoption. Several tools can help leaders in the process. ${ }^{*}$ Implementing a palliative approach often requires support from palliative experts outside the LTCH to build capacity within the LTCH. Unfortunately, the COVID-19 pandemic has halted many capacity-building activities, as infection control initiatives have taken precedence. This has limited, if not eliminated, support from the wider community for LTCHs to implement a palliative approach to care at a time when it is needed more than ever before. We strongly recommend that these activities are a priority, and that palliative consultants and specialists should be easily accessible.

Education should be provided to all staff, including personal support workers/care aides and, depending on the staffing model in the $\mathrm{LTCH}$, to the contract or consultant health-care providers (e.g., rehabilitation professionals, nurse practitioners, physicians). We recommend adopting one of the high-quality palliative education programs that would be appropriate for the individual LTCHs. ${ }^{\dagger}$ In addition to formal educational programs, other strategies, based on the Strengthening a Palliative Approach in Long Term Care Model (SPALTC) ${ }^{(12,13)}$ and Namaste Care, ${ }^{(14-16)}$ are recommended. Led by palliative nurse consultants or nurse practitioners, Comfort Care Rounds provide LTCH-wide, case-based discussions about deceased residents or those who are dying. ${ }^{(17)}$

\section{People With Dementia Should Be Engaged in ACP Early}

The pandemic has brought the critical need for ACP to the forefront. ACP is a "process that supports adults to understand and share their personal values, life goals, and preferences regarding future medical care to help ensure that people receive care that is consistent with their values, goals, and preferences during serious illness". ${ }^{(18)}$ In the LTCH context, ACP aims to activate critical communication early with residents and families to make decisions about EOL care. ${ }^{(19)}$ When ACP does not occur, family and care providers are not adequately prepared to make EOL decisions. They experience added stress and burden, with decisions being made in 'crisis mode'. Accommodating the challenges imposed by the pandemic so that residents participate in ACP activities should be a priority, given the evidence of positive health outcomes associated with ACP. ${ }^{(14,20-27)}$ We recommend using resources for residents, care partners, and clinicians that support holding ACP conversations in the early stages of dementia and in LTCHs experiencing COVID-19 outbreaks. Telehealth modes of participating in ACP during the pandemic are recommended. To be prepared, people with dementia should be engaged in ACP discussions (that should involve their family members/ significant others) to:

1. Choose a substitute decision-maker (SDM); and

2. Share with the SDM their values and wishes and what would matter most to them should they become seriously ill. ${ }^{(28)}$

\section{Family and Significant Others Should be Prepared and Supported for the End-of-Life}

Family members of people dying with dementia are vulnerable to stress, anxiety, social isolation, and decreased well-being, ${ }^{(29-34)}$ which has been exacerbated during the COVID-19 pandemic. Advanced dementia is a critical time for family involvement, given that involvement may impact the dying experience of the person with dementia and on the carer's bereavement and post-bereavement experience. ${ }^{(35-37)}$ Providing opportunities to develop and maintain meaningful relationships with a person with dementia during the pandemic is critical to minimize carer burden, stress, conflict, and loss.

To prepare families for EOL decision-making, it is critical that they are engaged in ACP discussions with residents early when they still have the capacity, so that when dementia has progressed, families are well informed and prepared to make decisions about goals of care on the residents' behalf. Given the growing demand for integration of a palliative approach to care within LTCHs due to the pandemic and COVID-19 outbreaks, and the increased awareness of service inadequacies due to visiting restrictions, virtual technologies offer a promising way to engage families and prepare them for decision-making when residents are nearing EOL and faceto-face discussions are not possible. At the very end of life, families need to know what to expect, including the signs of imminent death. They should be prepared for, and included in, a discussion about withdrawing or stopping treatments that are not helpful. Sitting vigil while a person is dying is a deeply ingrained norm, and dying alone is perceived as an indicator of a bad death by staff and families in LTCHs. ${ }^{(38)}$ Pandemicrelated visiting restrictions may limit deathbed vigils. Where possible, following local public health guidelines, family presence should be facilitated.

\section{End-of-Life Symptoms Should Be Managed Effectively}

Residents can present with various symptoms and complications throughout their illness journeys that may worsen at the EOL. These symptoms include dyspnea, pain, agitation and delirium, anxiety and depression, and airway secretions, amongst many others. Many, such as pain and delirium, are often under-recognized and mismanaged. ${ }^{(39-41)}$ Symptoms may progress gradually or, as with COVID-19 infection, progress rapidly, escalating within a few hours. Person-centred care approaches are needed, informed by, amongst other factors, the persons' life history and the clinical presentation. ${ }^{(42)}$ 
Optimal assessment and symptom management will reduce discomfort at the end-of-life and promote good death, ${ }^{(43)}$ and relieve some stress for family members who may not be able to visit due to pandemic-related restrictions.

LTCHs should build and ensure the capacity to optimally manage symptoms throughout the resident's illness and at the end of life when comfort measures take priority. ${ }^{(4,12,43,44,45-51)}$ Specific measures include:

- Adopting a proactive approach, including systematic and regular assessment of symptom severity and response to treatment, using validated and reliable tools; ${ }^{(52,53)}$ and considering pre-printed orders (PPO) such as for managing EOL symptoms ${ }^{(54,55)}$ and the dying protocol. ${ }^{(56)}$

- Providing access to palliative and geriatrics experts, not only to help build capacity but also to help manage complex and intractable symptoms. ${ }^{(42,57)}$

- Developing staff members' capacity to make rapid changes to care plans, and manage ethical dilemmas and challenges that may arise from conflicting values among or between staff and family members. For example, many people with advanced dementia may stop eating at the very EOL due to the inability to swallow. Staff must be able to differentiate between residents who cannot feed themselves from those who can no longer swallow, and offer comfort oral feeding that can curb the feelings of hunger and thirst. ${ }^{(58)}$ Families should be educated and supported to consider risks and benefits.

Staff in LTCHs should use the best evidence for the management of symptoms and complications related to advanced diseases and end-of-life. Clinical guidelines and protocols for the management of palliative symptoms and complications related to advanced diseases, ${ }^{* *}$ as well as care of patients with symptoms and complications caused by the COVID-19 disease, such as breathlessness, coughing, and delirium, are available. ${ }^{\dagger \dagger}$ Close collaboration is required between LTCHs and pharmacies to anticipate medication shortages, ${ }^{(59)}$ be proactive to prevent them where possible and, where they do occur, use alternative treatment approaches. LTCHs should also devise contingency plans where $24 / 7$ access to pharmacies are not available. LTCHs should have access to oxygen therapy and personal protective equipment (PPE), particularly in cases where certain therapies may risk aerosolizing the virus. ${ }^{(60)}$ It is essential that non-pharmacological management be incorporated wherever possible.

\section{After-Death Care Should Be Provided Consistently With Local Public Health Guidelines and Evidence About COVID-19 Transmission}

If the resident had confirmed or suspected diagnosis of COVID-19 infection, the LTCH should take specific measures informed by guidance from World Health Organization and their local public health authority. Though so far there is no evidence of persons contracting COVID-19 infection from being exposed to the bodies of an infected individual, it is important that LTCH staff follow routine practices such as hand hygiene, use of PPE during the last offices, and cleaning of equipment and environment when having cared for a person with confirmed or suspected COVID-19 disease. ${ }^{(61,62)} \mathrm{A}$ Point of Care Risk Assessment should be performed to gauge the risk of possible splatter from the resident's body fluids, in which case staff should don fluid resistant PPE. ${ }^{(63)}$ Policies and procedures should be in place that guide LTCH staff in taking utmost care to respect the dignity, religious, and cultural traditions of the residents while respecting and balancing rights of the family members. ${ }^{(61)}$ Finally, LTCHs should follow strict disinfecting and cleaning protocol, as human coronavirus can stay infectious for many days on surfaces. ${ }^{(64)}$

\section{Grief and Bereavement Support Should Be Provided to Family Members of Deceased Residents, LTCH Staff, and Surviving LTCH Residents, Including Residents With Dementia}

Probability of complicated or disenfranchised grief after the death of a LTCH resident during the pandemic is increased due to: (1) the unexpected nature of the death and rapid deterioration of residents who die from COVID-19; (2) inability to be with the resident and say goodbye; (3) the context of experiencing multiple other losses and stressors associated with the pandemic that tax coping and diminish resources and supports for coping; (4) potential disruption of faith and of religious and spiritual practices due to restrictions on gatherings and even being present with the person at death; (5) diminished social networks; and (6) lack of access to rituals, such as funerals, that help people process loss and grief. ${ }^{(65-69)}$

Support for residents with dementia should be individualized. It should not be assumed that people with dementia do not notice or are unaffected by deaths of people around them. (70,71) Rituals to mark the death of a resident are important for family, staff, and residents. These can include posting a memorial notice and photograph of the deceased resident; forming an honour guard when the body leaves the building; and recognition of the loss at the next meal through a moment of silence, words of remembrance, and a rose at the place of the deceased resident. ${ }^{(71)}$ Gatherings to mark residents' deaths could be replaced, while visiting is restricted, by virtual gatherings.

Support for bereaved families from LTCHs should include: (1) timely notification of the death; (2) expressing sympathy (card immediately and phone call to check in about a month after the death); (3) providing information about post-death services (e.g., funeral homes); and written information about grief and supports including potential disruption of grief because of pandemic restrictions, about the importance of drawing on their support networks and resources in their community.

Support for staff must acknowledge the intimate, often "family-like" relationships that care providers develop with residents and, as a result, their grief when residents die. Therefore, supervisors should acknowledge the meaning of the loss. Opportunities to express sympathy for, and say goodbye to, residents' family members are important. Staff rely on support from peers to process grief. This can be supported formally 
through debriefing sessions, and informal time to talk and reminisce following a death. Visiting restrictions increase risk for moral distress among grieving staff. In LTCHs that have COVID-19 outbreaks, the high numbers of deaths and potential for "bad deaths" also increase risk for moral distress and complicated bereavement. Hence, additional mental health supports should be available. ${ }^{(72)}$

These six recommendations are suitable for the residents of LTCHs throughout the globe. We hope that this position statement, commissioned by the Alzheimer Society of Canada and endorsed by a nationally representative multidisciplinary task force, will serve to be beneficial for optimizing care of older adults living with dementia in LTCH settings.

\section{ACKNOWLEDGEMENTS}

The authors thank the Alzheimer Society of Canada (ASC) for commissioning this work, as well as the members of the ASC COVID-19 and Dementia Task Force for their thoughtful comments on the draft manuscript. The authors also thank graduate students Danielle Just and Rachael Nalunga for assisting with manuscript preparation/references.

\section{CONFLICT OF INTEREST DISCLOSURES}

The authors declare that no conflicts of interest exist.

\section{ENDNOTES}

*For example, Kelley Model for Community Capacity Development. ${ }^{(73)}$

†Examples: (a) Pallium Canada's LEAP-LTC (https://www. pallium.ca/course/leap-long-term-care/), Online LEAP modules are currently offered free-of-charge during COVID-19;(74) (b) Foundations of Palliative Care Course for Frontline Staff, Centre for Education and Research on Aging and Health (http://cerah.lakeheadu.ca/palliative-care-for-front-lineworkers-/); (c) Quality Palliative Care in Long Term Care Toolkit (http://www.palliativealliance.ca/qpc-ltc-toolkit); (d) Fundamentals of Hospice Palliative Care (https://acclaim health.ca/programs/palliative-care-consultation/education/ fundamentals-of-hospice-palliative-care/)

ISpeak Up Canada. (n.d.). Advance Care Planning in Canada. https://www.advancecareplanning.ca/

'Speak Up Ontario. (2020). COVID-19 Resources. https:// www.speakupontario.ca/

** Palliative Care Guidelines: (a) Pallium Canada's Palliative Pocketbook and App ((https://www.pallium.ca/mobile-apppocketbook/); (b) Palliative EZ Guide, McMaster Palliative Care Symptom Management Guide and App Pocketbook and App (https://palliativeezguide.ca/); (c) British Columbia Centre for Palliative Care and Division of Palliative Care, University of British Columbia Guidelines (https://bc-cpc. ca/cpc/publications/symptom-management-guidelines/); (d) Fraser Health, British Columbia Palliative Care Guidelines (https://www.fraserhealth.ca/employees/clinical-resources/ hospice-palliative-care\#.XvJEsmhKjn0)

${ }^{\dagger}$ COVID-19 Palliative Care Guidelines: (a) McMaster Palliative Care COVID-19 Symptom Guidelines (Division of Palliative Care): Managing Dyspnea (https://fhs.mcmaster. $\mathrm{ca} /$ palliativecare/documents/McMasterDyspneaProtocol COVIDHamilton31March2020.pdf); (b) McMaster Palliative Care COVID-19 Symptom Guidelines (Division of Palliative Care): Other Symptoms (https://fhs.mcmaster.ca/palliativecare/documents/McMasterSymptomManagementProtocols COVID31March2020.pdf); (c) McMaster Palliative Care COVID-19 Symptom Guidelines (Division of Palliative Care): Palliative Sedation Pandemic Protocol (https://fhs.mcmaster. $\mathrm{ca} /$ palliativecare/documents/McMasterPalliativeSedationProtocolCOVID31March2020.pdf); (d) Fraser Health and Division of Palliative Care University of British Columbia Guidelines on Managing Dyspnea (https://bc-cpc.ca/cpc/wpcontent/uploads/2020/03/COVID-19-End-of-Life-SymptomManagement.pdf

\section{REFERENCES}

1. Government of Ontario. Ministry of Health. How Ontario is responding to COVID-19 [Internet]. Toronto, ON: Ontario Ministry of Health; 2020. Available from: https://www.ontario. ca/page/how-ontario-is-responding-covid-19

2. Van Der Steen JT. Dying with dementia: what we know after more than a decade of research. J Alzheimer's Dis. 2010;22(1):37-55.

3. De Gendt C, Bilsen J, Vander Stichele R, et al. Advance care planning and dying in nursing homes in Flanders, Belgium: a nationwide survey. J Pain Symptom Manage. 2013;45(2):223-34.

4. Hickman S, Unroe K, Ersek M, et al. Systematic advance care planning in the nursing home: Preliminary outcomes from the OPTIMISTIC (Optimizing Patient Transfers, Impacting Medical Quality, and Improving Symptoms: Transforming Institutional Care) demonstration project (S790). J Pain Symptom Manage. 2016;51(2):457-58.

5. Towsley GL, Hirschman KB. Talking about end-of-life care: perspectives of nursing home residents. Geriatr Nurs. 2018; 39(1):72-76.

6. Aronson L. (2020, March 28). Ageism is making the coronavirus pandemic worse [Internet]. The Atlantic. Retrieved from: https://www.theatlantic.com/culture/archive/2020/03/americasageism-crisis-is-helping-the-coronavirus/608905/

7. Pittis D. (2020, March 26). Politicians who consider sacrificing the old for the sake of the economy face a backlash: Don Pittis. CBC Online News. Retrieved from: https://www.cbc.ca/news/ business/coronavirus-covid-economy-seniors-1.5510079

8. Quality End of Life Care Coalition of Canada (QELCC). The way forward: an integrated palliative approach to care. Ottawa, ON: QELCC; 2020.

9. Van Der Steen JT, Radbruch L, Hertogh CM, et al. White paper defining optimal palliative care in older people with dementia: a Delphi study and recommendations from the European Association for Palliative Care. Palliat Med. 2014;28(3):197-209.

10. Collingridge Moore D, Payne S, Van den Block L, et al. Strategies for the implementation of palliative care education and organizational interventions in long-term care facilities: a scoping review. Palliat Med. 2020;34(5):558-70. 


\section{KAASALAINEN: END-OF-LIFE CARE FOR PEOPLE WITH DEMENTIA IN LTC}

11. Kaasalainen S. Current issues with implementing a palliative approach in long-term care: where do we go from here? Palliat Med. 2020;34(5):555-57.

12. Kaasalainen S, Sussman T, McCleary L, et al. Palliative care models in long-term care: A scoping review. Nurs Leadersh. 2019;32(3):8-26.

13. Kaasalainen S, Sussman T, McCleary L, et al. A Stakeholder Analysis of the Strengthening a Palliative Approach in LongTerm Care model. Nurs Leadersh. 2019;32(3):27-39.

14. Ashton SE, Roe B, Jack B, et al. End of life care: The experiences of advance care planning amongst family caregivers of people with advanced dementia - a qualitative study. Dementia. 2016;15(5):958-75.

15. Kaasalainen S, Hunter P V, Dal Bello-Haas V, et al. Evaluating the feasibility and acceptability of the Namaste Care program in long-term care settings in Canada. Pilot Feasibility Stud. 2020;6(1):34.

16. Bunn F, Lynch J, Goodman C, et al. Improving living and dying for people with advanced dementia living in care homes: a realist review of Namaste Care and other multisensory interventions. BMC Geriatr. 2018;18(1):303.

17. Wickson-Griffiths A, Kaasalainen S, Brazil K, et al. Comfort care rounds: a staff capacity-building initiative in long-term care homes. J Gerontol Nurs. 2015;41(1):42-48. Epub 2014 Jun 18.

18. Sudore RL, Lum HD, You JJ, et al. Defining advance care planning for adults: a consensus definition from a multidisciplinary Delphi panel. J Pain Symptom Manage. 2017;53(5):821-832.

19. Cantor MD, Pearlman RA. Advance care planning in long-term care facilities. J Am Med Dir Assoc. 2004;5(2 Suppl):S73-S80.

20. Black BS, Fogarty LA, Phillips H, et al. Surrogate decision makers' understanding of dementia patients' prior wishes for end-of-life care. J Aging Health. 2009;21(4):627-50.

21. Elliott BA, Gessert CE, Peden-Mcalpine C. Family decisionmaking in advanced dementia: narrative and ethics. Scand $J$ Caring Sci. 2009;23(2):251-58.

22. Brinkman-Stoppelenburg A, Rietjens JAC, Van Der Heide A. The effects of advance care planning on end-of-life care: a systematic review. Palliat Med. 2014;28(8):1000-25.

23. Chen CY, Thorsteinsdottir B, Cha SS, et al. Health care outcomes and advance care planning in older adults who receive home-based palliative care: a pilot cohort study. J Palliat Med. 2015;18(1):38-44.

24. Poppe M, Burleigh S, Banerjee S. Qualitative evaluation of Advanced Care Planning in Early Dementia (ACP-ED). PLoS One. 2013;8(4):e60412.

25. Radwany SM, Hazelett SE, Allen KR, et al. Results of the Promoting Effective Advance Care Planning for Elders (PEACE) randomized pilot study. Popul Health Manag. 2014;17(2):106-11.

26. Ke LS, Huang X, O'Connor M, et al. Nurses' views regarding implementing advance care planning for older people: a systematic review and synthesis of qualitative studies. JClin Nurs. 2015;24(15-16):2057-73.

27. Litzelman DK, Inui TS, Griffin WJ, et al. Impact of community health workers on elderly patients' advance care planning and health care utilization: moving the dial. Med Care. 2017;55(4):319-26.

28. Adraine Labs. The Conversation Project. Being prepared in the time of COVID-19: three things you can do now [Internet]. Boston, MA: Adraine Labs; 2020. Available from: https://theconversationproject.org/wp-content/uploads/2020/04/tcpcovid19guide. pdf
29. Chan D, Livingston G, Jones L, et al. Grief reactions in dementia carers: a systematic review. Int $J$ Geriatr Psychiatry. 2013;28(1):1-17.

30. Adelman RD, Tmanova LL, Delgado D, et al. Caregiver burden: a clinical review. JAMA. 2014;311(10):1052-60.

31. Bass DM, Judge KS, Lynn Snow A, et al. Negative caregiving effects among caregivers of veterans with dementia. Am J Geriatr Psychiatry. 2012;20(3):239-47.

32. Beinart N, Weinman J, Wade D, et al. Caregiver burden and psychoeducational interventions in Alzheimer's disease: a review. Dement Geriatr Cogn Dis Extra. 2012;2(1):638-48.

33. Papastavrou E, Kalokerinou A, Papacostas SS, et al. Caring for a relative with dementia: family caregiver burden. $J A d v$ Nurs. 2007;58(5):446-57.

34. Sörensen $\mathrm{S}$, Conwell Y. Issues in dementia caregiving: effects on mental and physical health, intervention strategies, and research needs. Am J Geriatr Psychiatry. 2011;19(6):491-96.

35. Romero MM, Ott CH, Kelber ST. Predictors of grief in bereaved family caregivers of person's with Alzheimer's disease: a prospective study. Death Stud. 2014;38(6):395-403.

36. Schulz R. Research priorities in geriatric palliative care: informal caregiving. J Palliat Med. 2013;16(9):1008-12.

37. Schulz R, Mendelsohn AB, Haley WE, et al. End-of-Life care and the effects of bereavement on family caregivers of persons with dementia. N Engl J Med. 2003;349(20):1936-42.

38. Thompson G, Shindruk C, Wickson-Griffiths A, et al. "Who would want to die like that?" Perspectives on dying alone in a long-term care setting. Death Stud. 2019;43(8):509-20.

39. Hall P, Schroder C, Weaver L. The last 48 hours of life in long-term care: a focused chart audit. J Am Geriatr Soc. 2002; 50(3):501-06.

40. Oliver DP, Porock D, Zweig S. End-of-life care in US nursing homes: a review of the evidence. J Am Med Dir Assoc. 2005;6 (3 Suppl):S21-S30.

41. Strumpf NE, Tuch H, Stillman D, et al. Implementing palliative care in the nursing home. Ann Long-Term Care. 2004;12(11):35-41.

42. Canadian Coalition for Seniors' Mental Health. Guideline on the assessment and treatment of delirium in older adults at the end of life [Internet]. Toronto, ON: CCSMH; 2010. Available from: https://ccsmh.ca/wp-content/uploads/2016/03/NatlGuideline_DeliriumEOLC.pdf

43. Thompson G, Wickson-Griffiths A. A palliative approach to care: from diagnosis to end of life. In: Garcia L, McCleary L, Drummond N, editors. Evidence-informed approaches for managing dementia transitions: riding the waves. Cambridge, MA: Academic Press; 2020. p. 213-34.

44. Registered Nurses Association of Ontario. End-of-life care during the last days and hours [Internet]. Toronto, ON: RNAO; 2011. Available from: https://rnao.ca/bpg/guidelines/endoflifecare-during-last-days-and-hours

45. Hall S, Goddard C, Stewart F, et al. Implementing a quality improvement programme in palliative care in care homes: a qualitative study. BMC Geriatr. 2011;11(1):31.

46. Parker D, Wilson J. The palliative approach toolkit [Internet]. Brisbane, Australia: University of Queensland; 2015.

47. Lee J, Cheng J, Au K, et al. Improving the quality of endof-life care in long-term care institutions. $J$ Palliat Med. 2013;16(10):1268-74.

48. Unroe KT, Nazir A, Holtz LR, et al. The Optimizing Patient Transfers, Impacting Medical Quality, and Improving Symp- 


\section{KAASALAINEN: END-OF-LIFE CARE FOR PEOPLE WITH DEMENTIA IN LTC}

toms: Transforming Institutional Care approach: preliminary data from the implementation of a Centers for Medicare and Medicaid Services nursing facility demonstration project. $J \mathrm{Am}$ Geriatr Soc. 2015;63(1):165-69.

49. Parker D, Hodgkinson B. A comparison of palliative care outcome measures used to assess the quality of palliative care provided in residential aged care facilities: a systematic review. JBI Evid Synthesis . 2010;8(3):90-120.

50. Ouslander G. IS6.10: Crossing the border of the nursing home - reducing unnecessary hospitalizations of vulnerable older patients: the INTERACT program. Eur Geriatr Med. 2014;5(Suppl 1):S10.

51. Vandenberg EV, Tvrdik A, Keller BK. Use of the quality improvement process in assessing end-of-life care in the nursing home. J Am Med Dir Assoc. 2006;7(3 Suppl):S82-S87.

52. Khosravani H, Steinberg L. Symptom management and endof-life care of COVID-19 residents in long-term care homes [blog post]. Canadian Family Physician. 2021. Available from: Symptom management and end-of-life care of COVID-19 residents in long-term care homes | The College of Family Physicians of Canada (cfp.ca)

53. McGuire DB, Kaiser KS, Haisfield-Wolfe ME, et al. Pain assessment in noncommunicative adult palliative care patients. Nurs Clin. 2016;51(3):397-431.

54. Stinson MJ, Gurevitz S, Carrigan A. Deprescribing at the end of life in older patients. J Am Acad Physician Assist. 2019;32(7):20-24.

55. Lavan AH, Gallagher P, Parsons C, et al. STOPPFrail (Screening Tool of Older Persons Prescriptions in Frail adults with limited life expectancy): consensus validation. Age Ageing. 2017;46(4):600-07.

56. Fraser Health Authority. Actively dying protocol pre-printed order and form [Internet]. Surrey, BC: The Authority; 2018. Available from: https:/www.fraserhealth.ca/employees/ student-practice-education/student-practice-news/activelydying-protocol-pre-printed-order-and-form\#.X05_KC0ZNTY

57. Cowan JD, Walsh D. Terminal sedation in palliative medicinedefinition and review of the literature. Support Care Cancer. 2001;9(6):403-07.

58. Arcand M. End-of-life issues in advanced dementia, Part 2: Management of poor nutritional intake, dehydration, and pneumonia. Can Fam Physician. 2015;61(4):337-41.

59. Pereira J, Downar J, Arya A, et al. Managing potential palliative care medication shortages during the COVID-19 pandemic: A guide provided by Pallium Canada [Internet]. Ottawa, ON: Pallium Canada; 2020. Available from: https://www.pallium. ca/wp-content/uploads/2020/05/Managing-Potential-PalliativeCare-Medication-Shortages-During-COVID-19.pdf

60. Pereira J, Barwich D, Nayar S. Managing dyspnea in patients with COVID-19 [webinar]. Ottawa, ON: Pallium Canada; 2020. Available from: https://www.pallium.ca/pallium-canadascovid-19-response-resources/

61. World Health Organization. Infection prevention and control for the safe management of a dead body in the context of COVID-19: interim guidance [Internet]. Geneva: WHO;
2020. Available from: https://www.who.int/publications/i/item/ infection-prevention-and-control-for-the-safe-management-ofa-dead-body-in-the-context-of-covid-19-interim-guidance

62. Public Health Agency of Canada. Routine practices and additional precautions for preventing the transmission of infection in healthcare settings [Internet]. Ottawa, ON: The Agency; 2017. Available from: https://www.canada.ca/en/public-health/ services/publications/diseases-conditions/routine-practicesprecautions-healthcare-associated-infections.html

63. BC Centre for Disease Control. Safe handling of bodies of deceased persons with suspected or confirmed COVID-19interim guidance [Internet]. Victoria, BC: BCCDC; 2020. Available from: http://www.bccdc.ca/Health-Professionals-Site/ Documents/COVID19_SafeHandlingBodies.pdf

64. Kampf G, Todt D, Pfaender S, et al. Persistence of coronaviruses on inanimate surfaces and their inactivation with biocidal agents. J Hosp Infect. 2020;104(3):246-51.

65. Bilgili GÖ. Mental health consequences of COVID-19 disaster. Infect Dis Clin Microbiol. 2020;2(1):42-45.

66. Wallace CL, Wladkowski SP, Gibson A, et al. Grief during the COVID-19 pandemic: considerations for palliative care providers. J Pain Symptom Manage. 2020;60(1):e70-e76.

67. Morris SE, Moment A, Thomas JD. Caring for bereaved family members during the COVID-19 pandemic: before and after the death of a patient. J Pain Symptom Manage. 2020;60(2):e70-e74.

68. Mayland CR, Harding AJE, Preston N, et al. Supporting adults bereaved through COVID-19: a rapid review of the impact of previous pandemics on grief and bereavement. J Pain Symptom Manage. 2020;60(2):e33-e39.

69. Moore KJ, Sampson EL, Kupeli N, et al. Supporting families in end-of-life care and bereavement in the COVID-19 Era. Int Psychogeriatr. 2020;32(10):1245-48.

70. Sussman T, Kaasalainen S, Mintzberg S, et al. Broadening end-of-life comfort to improve palliative care practices in long term care. Can J Aging. 2017;36(3):306-17.

71. McCleary L, Thompson GN, Venturato L, et al. Meaningful connections in dementia end of life care in long term care homes. BMC Psychiatry. 2018;18(1).

72. Maben J, Bridges J. Covid-19: supporting nurses' psychological and mental health. J Clin Nurs. 2020;29(15-16):2742-50.

73. Palliative Alliance. Quality palliative care in long term care: applying the Kelley Model for community capacity development [Internet]. Thunder Bay, ON: Lakehead University; 2017. Available from: https://www.palliativealliance.ca/applying-thekelley-model-for-community-capacity-development

74. Pereira J, Chary S, Moat JB, et al. Pallium Canada's curriculum development model: a framework to support large-scale courseware development and deployment. J Palliat Med. 2020;23(6):759-66.

Correspondence to: Sharon Kaasalainen, PhD, School of Nursing, McMaster University, 1280 Main Street West, HSC 3H48C, Hamilton, ON L8S 4K1

Email: kaasal@mcmaster.ca 